\title{
A median redshift of 2.4 for galaxies bright at submillimetre wavelengths
}

\author{
S. C. Chapman, ${ }^{*}$ A. W. Blain, ${ }^{\star}$ R. J. Ivison ${ }^{\dagger}$ and Ian Smail ${ }^{\ddagger}$ \\ * California Institute of Technology, Pasadena, CA 91125, USA \\ $\dagger$ Astronomy Technology Centre, Royal Observatory, Blackford Hill, \\ Edinburgh EH9 3HJ, UK \\ ¥ Institute for Computational Cosmology, University of Durham, South Road \\ Durham DH1 3LE, UK
}

\begin{abstract}
A significant fraction of the energy emitted in the early Universe came from very luminous galaxies that are largely hidden at optical wavelengths (because of interstellar dust grains); this energy now forms part of the cosmic background radiation at wavelengths near $1 \mathrm{~mm} .{ }^{1}$ These submillimetre (submm) galaxies were resolved from the background in $1997,{ }^{2}$ but have been difficult to identify and study due to the poor spatial resolution of submm instruments. ${ }^{3}$ This has impeded the determination of their distances (or redshifts, $z$ ), a crucial element in understanding their nature and evolution. ${ }^{4}$ Here we report spectroscopic redshifts for ten representative submm galaxies that we identified reliably using high resolution radio observations. ${ }^{5,6,7}$ The median redshift for our sample is 2.4, with a quartile range of $z=1.9-2.8$. The submm population therefore coexists with the peak activity of quasars, which are thought to be massive black holes in the process of accreting matter, suggesting a close relationship between the growth of massive black holes and luminous dusty galaxies. ${ }^{8}$ The space density of submm galaxies at $z>2$ is about 1000 times greater than that of similarly luminous galaxies in the present-day Universe, so they represent an important component of star formation at high redshifts.
\end{abstract}


The first real insight in the origin of the far-IR/submm background came with the commissioning of the SCUBA submm camera on the JCMT. Almost 100\% of the submm background has been resolved with the deepest SCUBA maps, which exploit a sensitivity boost from gravitational lenses. ${ }^{2,3,9}$ However, the near-IR/optical faintness of the submm galaxy population conspires with the modest positional precision available from the large $\left(15^{\prime \prime}\right)$ SCUBA beam and the large surface density of unrelated optically-faint galaxies to render positional coincidence alone inadequate to identify counterparts. ${ }^{3,5}$ This problem is compounded by the small field of view of SCUBA: compiling large samples of submm galaxies is slow, with roughly one detected every night. Because of these difficulties, robust spectroscopic redshifts have been published for only a handful of submm sources, all atypically bright at optical wavelengths. ${ }^{10,11,12,3}$

Here, we have overcome these problems by taking advantage of $1.4-\mathrm{GHz}$ radio data from the Very Large Array (VLA) radio telescope (Hubble Deep Field ${ }^{13}$, SSA13 field ${ }^{14}$, ElaisN2 and Lockman fields $\left.{ }^{7}\right)$. These deep images have fine $\left(1-2^{\prime \prime}\right)$ spatial resolution and a large ( $30^{\prime}$ FWHM) field of view. The radio data was exploited to followup SCUBA submm data in the Hubble Deep Field (12 hr RA) and SSA13 (13 hr RA) fields ${ }^{15,5,16}$, and the Lockman (10 hr RA) and ElaisN2 (16 hr RA) fields ${ }^{17}$. The radio data are sensitive to the synchrotron radio emission from cosmic-ray electrons accelerated in the supernovae explosions of the same high-mass stars that heat the dust and generate the far-IR/submm emission. Hence a deep radio image should be an efficient route to pinpoint the positions of many submm galaxies. This is confirmed in fields with both submm and radio observations: radio counterparts brighter than $30 \mu \mathrm{Jy}$ can be found for $60-70 \%$ of submm galaxies brighter than $5 \mathrm{mJy}$ at $850 \mu \mathrm{m}$ (which have a surface density of $450 \mathrm{deg}^{-2}, 3$ and contribute about $20 \%$ of the submm background). ${ }^{7}, 16$ Moreover, the accurate radio positions mean that SCUBA's efficient 'photometry' mode can be used to search for submm galaxies, ${ }^{5,6,18}$ raising the detection rate to around ten per night.

Although submm galaxies have faint optical continuum emission, ${ }^{3}$ the radio-detected samples are sufficiently large to allow efficient, multi-object optical spectroscopy using 10-m telescopes. Slit positions can be assigned accurately, as the radio/submm emission can be 
located on optical images to within $0.3-0.8^{\prime \prime} .{ }^{7}$ Using this approach we targetted 34 radiodetected submm galaxies mostly brighter than $5 \mathrm{mJy}$ at $850 \mu \mathrm{m}$ with optical magnitudes in the range $I=22.2-26.4$ using the ESI and LRIS spectrographs on the Keck telescopes (Fig. 1). We measured redshifts for ten submm galaxies, representative of the blank-field population. We have doubled the number of optically bright $(I<23.5)$ submm galaxies with accurate redshifts. Our six redshifts for $I>23.5$ submm galaxies represent the first redshifts for this class of submm galaxy. The redshifts span the range $z=0.8-3.7$, with a median of 2.4 and an interquartile range of 1.9-2.8 (Fig. 2). These redshifts allow dust temperatures $T_{\mathrm{d}}$ and bolometric luminosities to be determined (see Table 1), assuming that the tight correlation between far-IR and radio emission observed at low redshifts ${ }^{19}$ remains valid. Typical values are of order $35 \mathrm{~K}$ and several $10^{12} \mathrm{~L}_{\odot}$ respectively. Note that relativistic electrons accelerated in shocks close to an active galactic nucleus (AGN) should boost the radio flux density above that expected from the standard correlation. If the radio emission is boosted by an AGN then both temperature and luminosity are overestimated. There are no obvious cases in the present sample in this category.

The redshifts impose robust limits to the space densities of submm galaxies in the redshift ranges $0.5-1.2,1.8-2.8$ and $2.8-4$ (the gap at $z=1.2-1.8$ is the spectroscopic desert in which no strong restframe UV lines are redshifted into the optical window): we calculate $\rho=(3.3 \pm 2.3) \times 10^{-6} \mathrm{Mpc}^{-3},(6.5 \pm 2.5) \times 10^{-6} \mathrm{Mpc}^{-3}$ and $(2.4 \pm 1.2) \times 10^{-6}$ $\mathrm{Mpc}^{-3}$, respectively, assuming a flat $\Lambda=0.7$ Universe with $H_{0}=65 \mathrm{~km} \mathrm{~s}^{-1} \mathrm{Mpc}^{-1}$. This compares to a space density of $\sim 10^{-8} \mathrm{Mpc}^{-3}$ for low-redshift galaxies with comparable bolometric luminosities greater than $5 \times 10^{12} \mathrm{~L}_{\odot}$, comparable to the luminosities of our spectroscopically targeted galaxies at any redshift greater than one. The volume density of very luminous galaxies thus increases almost a thousandfold from $z \sim 0$ to 2 .

The relationship between different classes of high-redshift galaxy is critical for our understanding of galaxy evolution. Our spectroscopy shows that the submm galaxies are coeval with the important populations of star-forming galaxies and quasars detected at $z=$ 2-3 using optical and X-ray techniques. . $^{80,21,22}$ We can use their relative space densities to compare and relate these populations. In a $1000 \mathrm{Mpc}^{3}$ box at $z \sim 2.5$, there are 10 Lyman- 
break galaxies with $R_{\mathrm{AB}}<25.5\left(\sim 0.2 \times \mathrm{L}_{1500}^{*}\right)$ and 1 submm galaxy from our sample (with $850 \mu \mathrm{m}$ flux $>5 \mathrm{mJy}$ ), but the single submm galaxy produces a comparable luminosity to the 10 Lyman-break galaxies. (Here $R_{\mathrm{AB}}$ is the flux normalized magnitude near $6900 \AA$; $L_{1500}^{*}$ is the characteristic luminosity at restframe $1500 \AA$, denoting a change in slope in the luminosity function.) Equally, the volume density of submm galaxies is similar to that of narrow-line Type-II AGN selected at these redshifts, from both X-ray and optical surveys, and an order of magnitude greater than that of bright optical quasars with $M_{B}>-25 .{ }^{8}$ However, the links between the different classes of sources all depend critically on whether the activity is intermittent or continuous.

To quantify the whole submm population using this survey we must account for several selection effects. In particular, requiring a radio identification prior to spectroscopy limits the maximum redshift. Studies of galaxies at low and moderate redshifts suggest that changes in the dust properties, especially the dust temperature $T_{\mathrm{d}}$, modify the relative strength of the emission in the submm and radio wavebands. ${ }^{23}$ The detectability of distant submm galaxies (at a fixed 850- $\mu \mathrm{m}$ flux) in the radio waveband thus depends on the dust temperature: warmer galaxies are detectable at $z \geq 3$, while the radio emission from cooler sources falls below the flux limit at lower redshifts. ${ }^{18}$ About $30 \%$ of submm galaxies that are brighter than $5 \mathrm{mJy}$ at $850 \mu \mathrm{m}$ are not detected in the radio: these galaxies would be lost from our catalogue at $z \sim 2.5$ for the coldest plausible $T_{\mathrm{d}} \sim 25 \mathrm{~K}$, and at $z>3$ for the typical $T_{\mathrm{d}} \simeq 35 \mathrm{~K}$ that we find. Hence, we can only place a lower limit on the space density in the highest redshift interval. The density derived at $z=2$ should be unaffected by the need for the galaxies to be detected at radio wavelengths.

We must also consider the completeness of our spectroscopic observations. While our redshift completeness is relatively high $(\sim 30 \%)$, we failed to find convincing redshifts for 24 galaxies. However, nine of of these have strong single emission line detections which could plausibly be identified as Lyman- $\alpha$. Some of the remainder without emission lines could lie in the spectroscopic desert, making redshifts difficult to identify. The absence of sources at $z \sim 1.5$ in Table 1 suggests that this effect is probably responsible for some of the incompleteness. Our targets generally have faint UV continua, and so there is a bias 
towards identifying redshifts for galaxies with strong emission lines. If the missing galaxies lie outside of the desert, but lack strong emission lines, then their radio detection suggests they probably lie in the same $z=1-4$ range spanned by the spectroscopically identified sources.

On the basis of bolometric luminosities estimated from our radio and submm measurements and redshifts (Table 1), assuming the far-IR-radio correlation, we calculate the following luminosity densities from our survey: $\rho_{\mathrm{L}}(0.5<z<1.2)=(5 \pm 5) \times 10^{6} \mathrm{~L}_{\odot} \mathrm{Mpc}^{-3}$, $\rho_{\mathrm{L}}(1.8<z<2.8)=\left(7_{-4}^{+6}\right) \times 10^{7} \mathrm{~L}_{\odot} \mathrm{Mpc}^{-3}$ and $\rho_{\mathrm{L}}(2.8<z<4.0)=\left(7_{-5}^{+10}\right) \times 10^{7} \mathrm{~L}_{\odot} \mathrm{Mpc}^{-3}$. To translate these values into star-formation densities we must subtract any contribution to the luminosities of these galaxies from the heating of dust by an active galactic nucleus (AGN). The proportion of the submm population detected in deep Chandra and XMMNewton X-ray surveys, which are sensitive to even dust-obscured AGN, ${ }^{7,20}$ at flux densities significantly greater than that expected from star formation alone is at most $30 \%$. Hence, we assume conservatively that the far-IR luminosity is derived from star formation in the remaining 70\%. Luminosity-density measurements can be translated into a star-formation density using the standard calibration of $1.6-2.2 \times 10^{9} \mathrm{~L}_{\odot}\left(\mathrm{M}_{\odot} \mathrm{yr}^{-1}\right)^{-1} .{ }^{24}$

The bright radio-detected submm galaxies presented here represent just $20 \%$ of the 850- $\mu \mathrm{m}$ background, yet their estimated star-formation densities at redshifts of 2 and 3 are comparable with those inferred from optical observations. ${ }^{21}$ Both of these estimates require corrections, in the case of the optical surveys an increase of a factor of 5 is needed to account for dust obscuration, ${ }^{25}$ while our submm estimate needs to be corrected by a similar factor to account for spectroscopic incompleteness and sources below our submm flux limit. We find that the submm and Lyman-break populations encompass all of the star formation activity needed to reproduce the star formation history inferred from models of the background radiation and submm counts. ${ }^{4}$ Hence, our substantially complete redshift survey provides the strongest evidence for a rapid increase in the volume density of dusty, very luminous galaxies in the distant Universe. Their density peaks at a redshift $z \simeq 2.4$ at a value about 1000 times greater than that at the present epoch. Thus we conclude that the balance between the star-formation density in the most luminous dust-obscured versus 
unobscured galaxies has altered radically over the last $80 \%$ of the history of the Universe. 


\section{References.}

1. Fixsen, D. J., Dwek, E., Mather, J. C., Bennett, C. L., Shafer, R. A. The spectrum of the extragalactic far-infrared background from the COBE FIRAS observations. Astrophys. J. 508, 123-128 (1998).

2. Smail, I., Ivison, R. J., \& Blain, A. W. A deep submillimeter survey of lensing clusters: a new window on galaxy formation and evolution. Astrophys. J. 490, L5-L8 (1997).

3. Smail, I., Ivison R. J., Blain, A. W., \& Kneib, J.-P. The nature of faint submillimetreselected galaxies. Mon. Not. R. Astron. Soc. 331, 495-520 (2002).

4. Blain, A. W., Smail, I., Ivison, R. J., Kneib, J.-P. The history of star-formation in dusty galaxies. Mon. Not. R. Astron. Soc. 302, 632-648 (1999).

5. Barger, A. J., Cowie, L. L., Richards, E. A. Mapping the evolution of high-redshift dusty galaxies with submillimeter observations of a radio-selected sample. Astron. J. 119, 2092-2109 (2000).

6. Chapman, S. C., Richards, E. A., Lewis, G. F., Wilson, G., Barger, A. J. The nature of the bright submillimeter galaxy population. Astrophys. J. 548, L147-L151 (2001).

7. Ivison, R. J., Greve, T., Smail, I., Dunlop, J., Roche, N., Scott, S., Page M., Stevens, J., Almaini, O., Blain, A., Willott, C., Fox, M., Gilbank, D., Serjeant, S., \& Hughes D. Deep radio imaging of the SCUBA 8-mJy survey fields: submm source identifications and redshift distribution. Mon. Not. R. Astron. Soc. 337, 1 (2002).

8. Boyle, B. J., Shanks, T., Croom, S. M., Smith, R. J., Miller, L., Loaring, N., Heymans, C. The 2dF QSO redshift survey - I. The optical luminosity function of quasi-stellar objects. Mon. Not. R. Astron. Soc. 317, 1014-1022 (2000).

9. Cowie, L. L., Barger, A. J., Kneib, J.-P. Faint Submillimeter Counts from Deep 850 Micron Observations of the Lensing Clusters A370, A851, and A2390. Astron. J. 123, 2197-2205 (2002).

10. Ivison, R. J., Smail, I., Le Borgne, J.-F., Blain, A. W., Kneib, J.-P., Bezecourt, J., Kerr, T. H., \& Davies, J. K. A hyperluminous galaxy at $z=2.8$ found in a deep submillimetre survey. Mon. Not. R. Astron. Soc. 298, 583-593 (1998). 
11. Barger, A. J., Cowie, L. L., Smail, I., Ivison, R. J., Blain, A. W., \& Kneib, J.-P. Redshift distribution of the faint submillimeter galaxy population. Astron. J. 117, 2656-2665 (1999).

12. Ivison, R. J., Smail, I, Barger, A. J., Kneib, J.-P., Blain, A. W., Owen, F. N., Kerr, T. H., \& Cowie, L. L. The diversity of SCUBA-selected galaxies Mon. Not. R. Astron. Soc. 315, 209-221 (2000).

13. Richards, E. A. The Nature of Radio Emission from Distant Galaxies: The 1.4 GHZ Observations. Astrophys. J. 533, 611-630 (2000).

14. Fomalont, E. B., Kellermann, K. I., Partridge, R. B., Windhorst, R. A., Richards, E. A. The Microjansky Sky at 8.4 GHz. Astron. J. 123, 2402-2416 (2002).

15. Barger, A. J., Cowie, L. L., Sanders, D., Fulton, E., Taniguchi, Y., Sato, Y., Kawara, K., Okuda, H. Submillimetre-wavelength detection of dusty star-forming galaxies at high redshift. Nature 394, 248-251 (1998).

16. Chapman, S. C., Barger, A., Cowie, L., Borys, C., Capak, P., Fomalont, E., Lewis, G., Richards, E., Scott, D., Steffen, A., Wilson, G., Yun, M., The properties of microjansky radio sources in the HDF-N, SSA13, and SSA22 Fields. Astrophys. J. 585, 57 (2003).

17. Scott, S. E., Fox, M. J., Dunlop, J. S., Serjeant, S., Peacock, J. A., Ivison, R. J., Oliver, S., Mann, R. G., Lawrence, A., Efstathiou, A., Rowan-Robinson, M., Hughes, D. H., Archibald, E. N., Blain, A., Longair, M. The SCUBA 8-mJy survey - I. Submillimetre maps, sources and number counts. Mon. Not. R. Astron. Soc. 331, 817-837 (2002).

18. Chapman, S. C., Lewis, G. F., Scott, D., Borys, C., Richards, E.A., Understanding the nature of the optically faint radio sources and their connection to the submillimeter population. Astrophys. J. 570, 557-570 (2002).

19. Condon, J. Radio emission from ordinary galaxies. Annu. Rev. Astron. Astrophy. 30, 575-611 (1992).

20. Barger, A. J., Cowie, L. L., Brandt, W. N., Capak, P., Garmire, G. P., Hornschemier, A. E., Steffen, A. T., Wehner, E. H. X-ray, optical and infrared imaging and spectral 
properties of the 1-Ms Chandra Deep Field North sources. Astron. J. 124, 1839 (2002).

21. Steidel, C. C., Adelberger, K. L., Giavalisco, M., Dickinson, M. \& Pettini, M. Lymanbreak galaxies at $z>4$ and the evolution of the ultraviolet luminosity density at high redshift. Astrophys. J. 519, 1-17 (1999).

22. Steidel, C., Hunt, M., Shapley, A., Adelberger, K., Pettini, M., Dickinson, M. \& Giavalisco, M. The population of faint optically-selected AGN at $z \sim 3$. Astrophys. $J$. in the press; also available as prerint astro-ph/0205142 at http://www.arXiv.org (2002).

23. Blain, A. W., Smail, I., Ivison, R. J., Kneib, J.-P., Frayer, D. T. Submillimeter galaxies. Physics Reports 369, 111-196; also available as preprint astro-ph/0202228 at http://www.arXiv.org (2002).

24. Kennicutt, R. C. Star formation in galaxies along the Hubble sequence. Annu. Rev. Astron. Astrophys. 36, 189-232 (1998).

25. Pettini, M., Shapley, A. E., Steidel, C. C., Cuby, J.-G., Dickinson, M., Moorwood, A. F. M., Adelberger, K. L., \& Giavalisco, M. The rest-frame optical spectra of Lymanbreak galaxies: star formation, extinction, abundances and kinematics. Astrophys. J. 554, 981-1000 (2001).

26. Chapman, S. C., Helou, G., Lewis, G. F., Dale, D., The bi-variate luminosity-color distribution of IRAS galaxies, and impications for the high redshift Universe. Astrophys. $J$. in the press;also available as preprint astro-ph/0301233 at http://www.arXiv.org (2002).

27. Chapman, S. C., Smail, I., Ivison, R., Blain, A., The effect of lensing on the identification of SCUBA galaxies, Mon. Not. R. Astron. Soc. 335, 17-21 (2002).

28. Oke, J. B., Cohen, J. G., Carr, M., Cromer, J., Dingizian, A., Harris, F. H., Labrecque, S., Lucinio, R., \& Schaal, W. The Keck low-resolution imaging spectrometer. Publ. Astron. Soc. Pac. 107, 375-385 (1995). 
29. Sheinis, A. I., Bolte, M., Epps, H. W., Kibrick, R. I., Miller, J. S., Radovan, M. V., Bigelow, B. C., \& Sutin B. M. ESI, a new Keck Observatory echellette spectrograph and imager. Publ. Astron. Soc. Pac. 114, 851-865 (2002).

30. Smail, I., et al., A vigorous starburst in the SCUBA galaxy N2-850.4. Mon. Not. R. Astron. Soc. (in the press); preprint available at http://www.arXiv.org/astroph/0303128 (2003).

\section{Acknowledgments.}

We are grateful to Chuck Steidel, Alice Shapley and Tim Heckman for insightful discussions. SCC acknowledges support from NASA. IRS acknowledges support from the Royal Society and a Philip Leverhulme Prize Fellowship. NRAO is operated by Associated Universities Inc., under a cooperative agreement with the US National Science Foundation. Data presented herein were obtained using the W. M. Keck Observatory, which is operated as a scientific partnership among Caltech, the University of California and NASA. The Observatory was made possible by the generous financial support of the W. M. Keck Foundation.

The authors declare that they have no competing financial interests.

Correspondence and requests for materials should be addressed to Scott Chapman (schapman@irastro.caltech.edu). 
Table 1. Positions of submm galaxies with redshifts from our survey, along with their measured redshifts, submm fluxes and optical magnitudes, infered dust temperatures $T_{\mathrm{d}}$ and bolometric luminosities $L_{\mathrm{bol}} \cdot T_{\mathrm{d}}$ and $L_{\mathrm{bol}}$ were calculated, based on the redshift, submm and radio flux densities, assuming that the far-IR-radio correlation applies. Sources are in order of decreasing flux density, as in Figure 1. (SMM163704.3+410530 was previously published in ${ }^{27}$.) Submm fluxes for sources in the Lockman (10 hr RA) and ElaisN2 (16 hr RA) fields are from ${ }^{17}$ while fluxes for other sources were measured ourselves. Six out of 10 galaxies have $I$-band magnitudes fainter than 23.5, consistent with the optical flux distribution of blank-field submm galaxies presented elsewhere, ${ }^{16}$ and confirming that our sample is representative of the population. Galaxies with hotter inferred dust temperatures could have their radio flux densities enhanced by contribution from an active galactic nucleus. Understanding the relationship between submm galaxies and other high-redshift populations will be helped by comparing the strength of the clustering, both within and between the different classes. This will require substantially larger samples than are currently available.

\begin{tabular}{|c|c|c|c|c|c|}
\hline dec & redshift & $\mathrm{S}_{850 \mu m}(\mathrm{r}$ & $I$-mag & $\mathrm{T}_{d}(\mathrm{~K})$ & $\mathrm{L}_{\text {bol }}\left(10^{12} \mathrm{~L}_{\odot}\right)$ \\
\hline SMM105224.6 +572119 & 2.429 & $11.7 \pm 3.4$ & 25.9 & $21 \pm 5$ & $3.0_{-0.7}^{+2.0}$ \\
\hline SMM163704.3 +410530 & 0.840 & $11.2 \pm 1.6$ & 21.5 & $16_{-2.2}^{+4.1}$ & $0.58_{-.34}^{+.32}$ \\
\hline SMM105230.6 +572212 & 2.610 & $11.0 \pm 2.6$ & 23.0 & $29 \pm 6$ & $6.9_{-3.3}^{+5.9}$ \\
\hline SMM163650.0 +405733 & 2.376 & $8.2 \pm 1.7$ & 22.2 & $54_{-7}^{+11}$ & $45_{-19}^{+53}$ \\
\hline SMM123600.2 +621047 & 1.998 & $7.9 \pm 2.4$ & 23.6 & $37_{-4}^{+8}$ & $10_{-4}^{+11}$ \\
\hline SMM131201.2 +424208 & 3.419 & $6.2 \pm 1.2$ & 23.5 & $48_{-7}^{+10}$ & $20_{-7}^{+18}$ \\
\hline SMM105207.7 +571907 & 2.698 & $6.2 \pm 1.6$ & 26.0 & $36_{-4}^{+7}$ & $7.8_{-2.3}^{+7.1}$ \\
\hline SMM131212.7 +424423 & 2.811 & $5.6 \pm 1.9$ & 26.4 & $54_{-10}^{+19}$ & $30_{-16}^{+60}$ \\
\hline SMM163658.8 +405733 & 1.189 & $5.1 \pm 1.4$ & 22.4 & $25_{-9}^{+5}$ & $1.5 \pm 1.2$ \\
\hline SMM105155.7 +572312 & 3.699 & $4.5 \pm 1.3$ & 24.2 & $59_{-16}^{+26}$ & $30_{-20}^{+82}$ \\
\hline
\end{tabular}




\section{Figure Legends.}

Figure 1. The ten spectra from Table 1 with identified redshifts (smoothed to the instrumental resolution) from the sample of 34 submm galaxies observed, spanning the $I=22.2-26.4$ range. (Note that SMM163704.3+410530 was previously published in ${ }^{27}$.) Pilot observations, using Keck's ESI ${ }^{29}$ spectrograph on 2001 July 16 in the ELAIS-N2 ${ }^{17}$ field yielded three spectra with strong emission lines. Multiobject Keck observations of 31 submm galaxies using LRIS $^{28}$ were made on 2002 March 18-19 in light cirrus conditions (with good $0.8^{\prime \prime}$ seeing) in the HDF \& SSA13 fields ${ }^{5,16}$, and the Lockman Hole and ELAIS-N2 fields ${ }^{17}$. All the spectra probe the observed wavelength range from $0.3-1 \mu \mathrm{m}$. Exposures times were 1.5-4.5 hr, split into 30-min integrations. Data reduction followed standard techniques using custom IRAF scripts. One-dimensional spectra were extracted and compared with template spectra and emissionline catalogues to identify redshifts. All identifications are based on multiple lines, most prominently the Ly $\alpha$ line which varies tremendously in both flux (ranging from 1 to $60 \mu \mathrm{Jy}$ ) and rest-frame equivalent

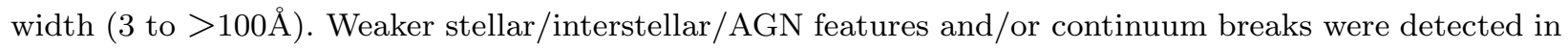
the spectra, strengthening the redshift identifications. Two galaxies at the same redshifts were identified in the vicinity of the 10 robust submm sources, as shown for the companions SMMJ105155.7+572312-a (one of the few submm galaxies showing Lyman- $\alpha$ in absorption) and SMMJ105155.7+572312-b. The highest S/N spectrum (SMMJ163650.0+405733 ${ }^{30}$ ) includes features indicative of both strong starburst activity (P-Cygni wind absorption profiles, stellar/interstellar absorption lines) and a weak AGN, a mix typical of hybrid starburst/Seyfert-2 galaxies. None of our spectra have broad lines like Type-I AGN. Narrow-line Type-II AGN with enhanced N V and/or C IV emission are consistent with half of our spectra. Despite this evidence for AGN in these galaxies, they appear to be relatively weak in energetic terms as compared to those identified in X-ray or optical surveys.

Figure 2. The redshift histogram of our submm galaxy sample. We describe the selection effects using an evolving model of the local far-IR luminosity function, in which the dusty galaxies are represented by a range of template spectral energy distributions. The model has been tuned to fit the statistical properties of the submm-radio galaxy population (surface density on the sky and submm-radio colours). ${ }^{26}$ As a range of spectral energy distributions are represented at each galaxy luminosity, a broader redshift distribution results than if the luminosity was tied one-to-one with the dust temperature. ${ }^{23}$ We plot the predicted redshift distributions for submm galaxies with flux densities $S_{850 \mu m}>5 \mathrm{mJy}$ (solid line) and 
radio sources with $30 \mu \mathrm{Jy}<S_{1.4 \mathrm{GHz}}<500 \mu \mathrm{Jy}$ (dashed line). We expect to miss sources lying between the submm and radio model curves due to our requirement of a radio detection to pinpoint the submm source. The apparent deficit between model and data at $z \sim 1.5$ may be indicative of the difficulty in obtaining spectra in the spectroscopic desert that spans the range $1.2<z<1.8$. Note that the models are in good overall agreement with the observed redshift distribution. 

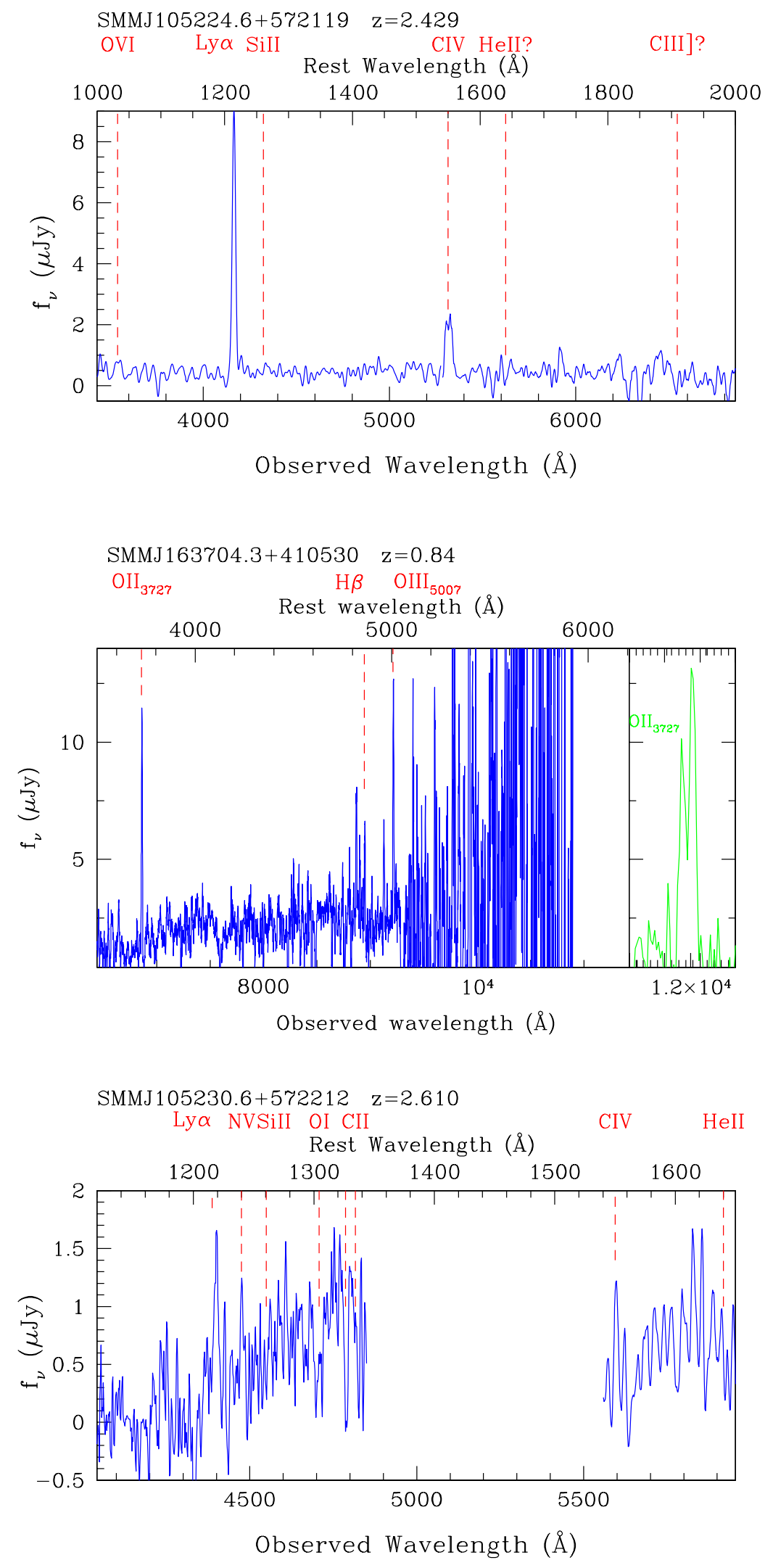

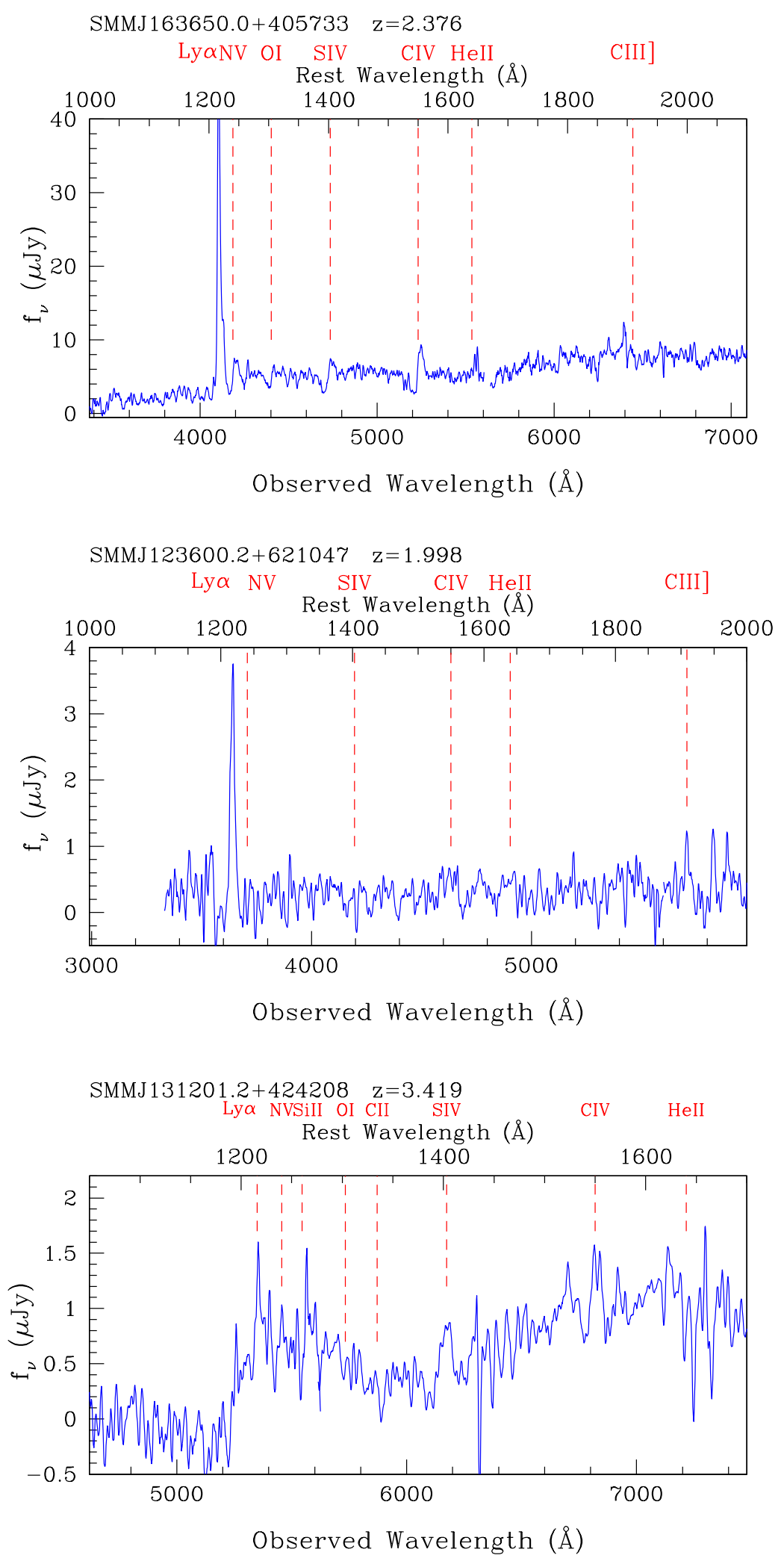

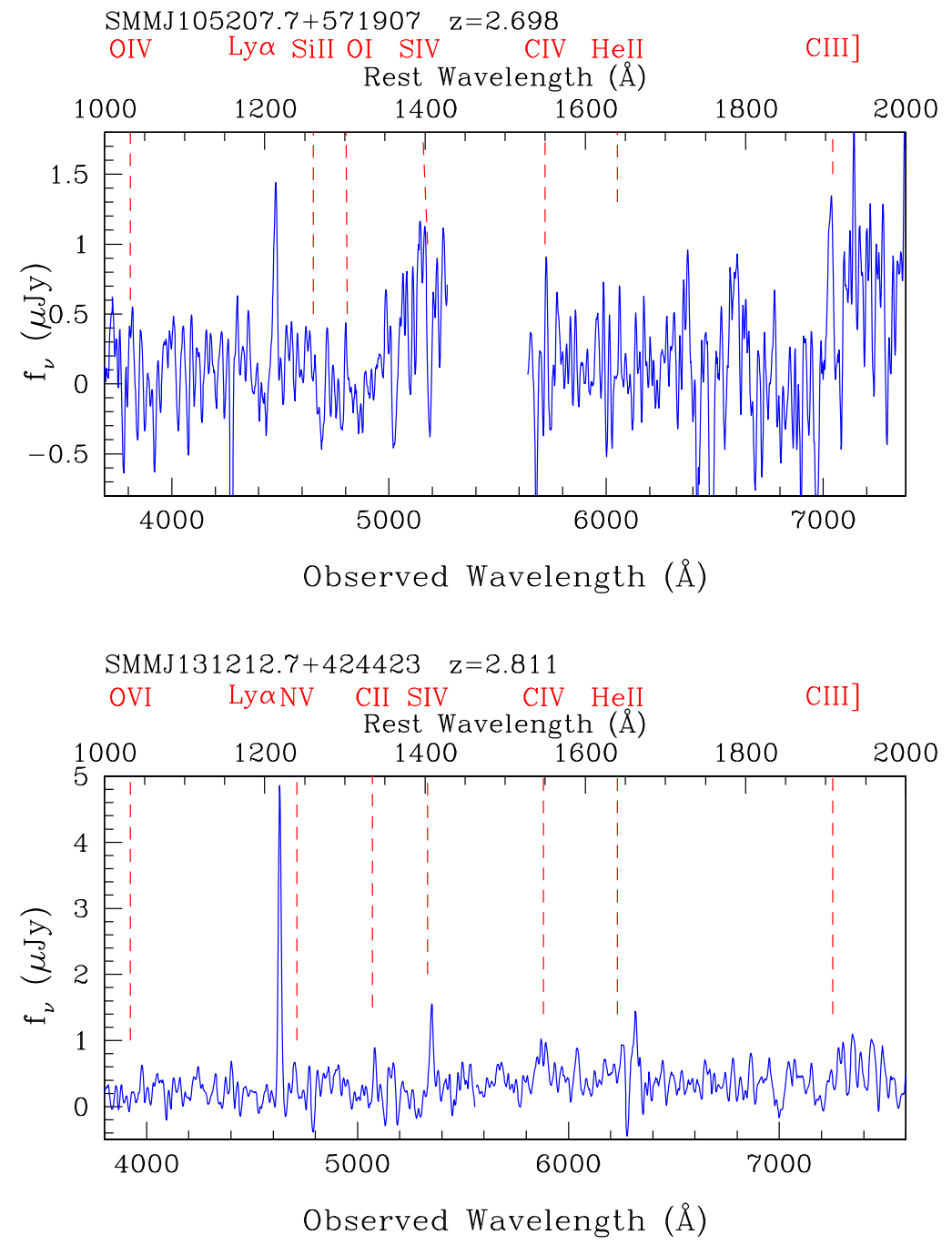

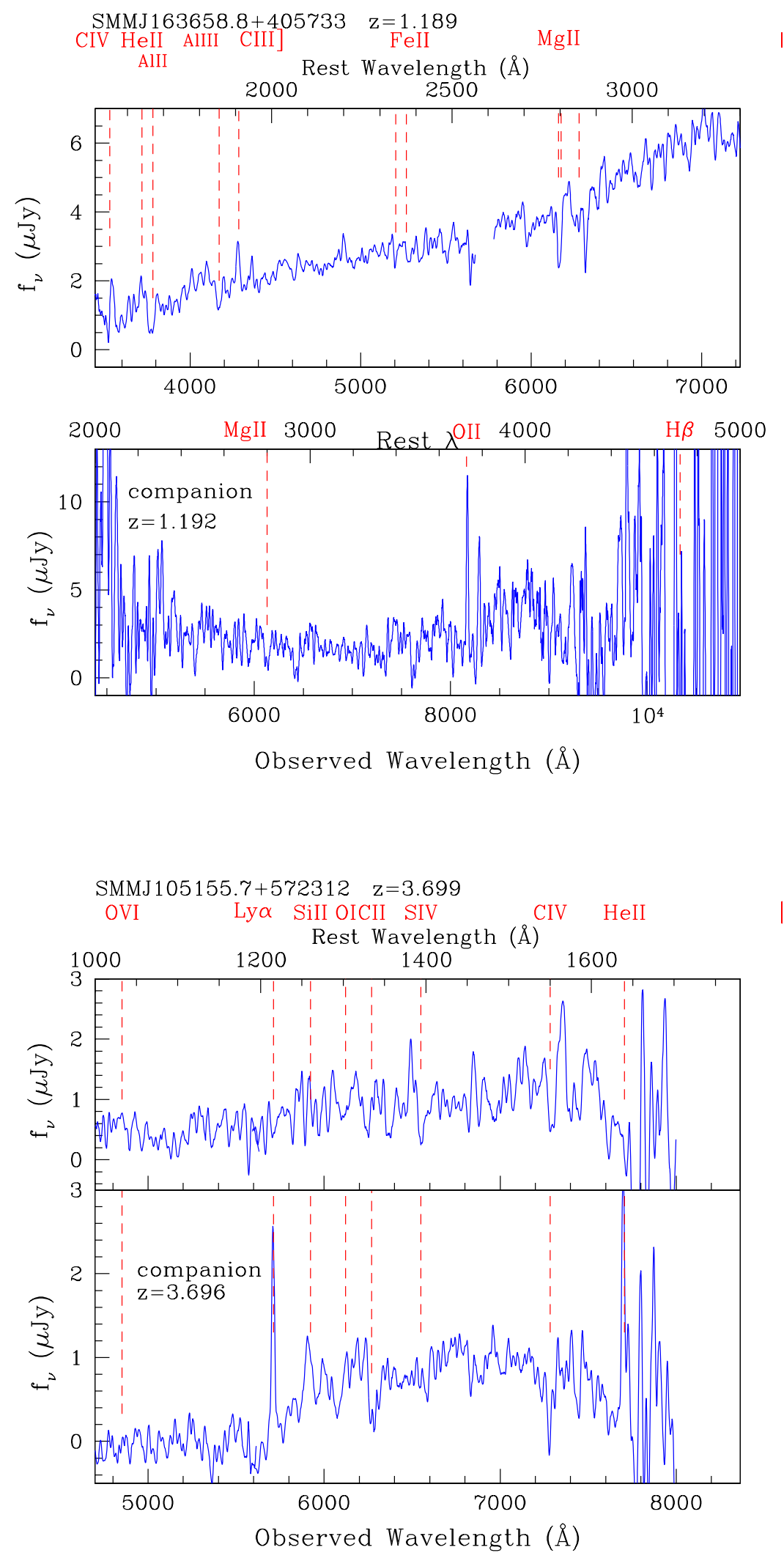


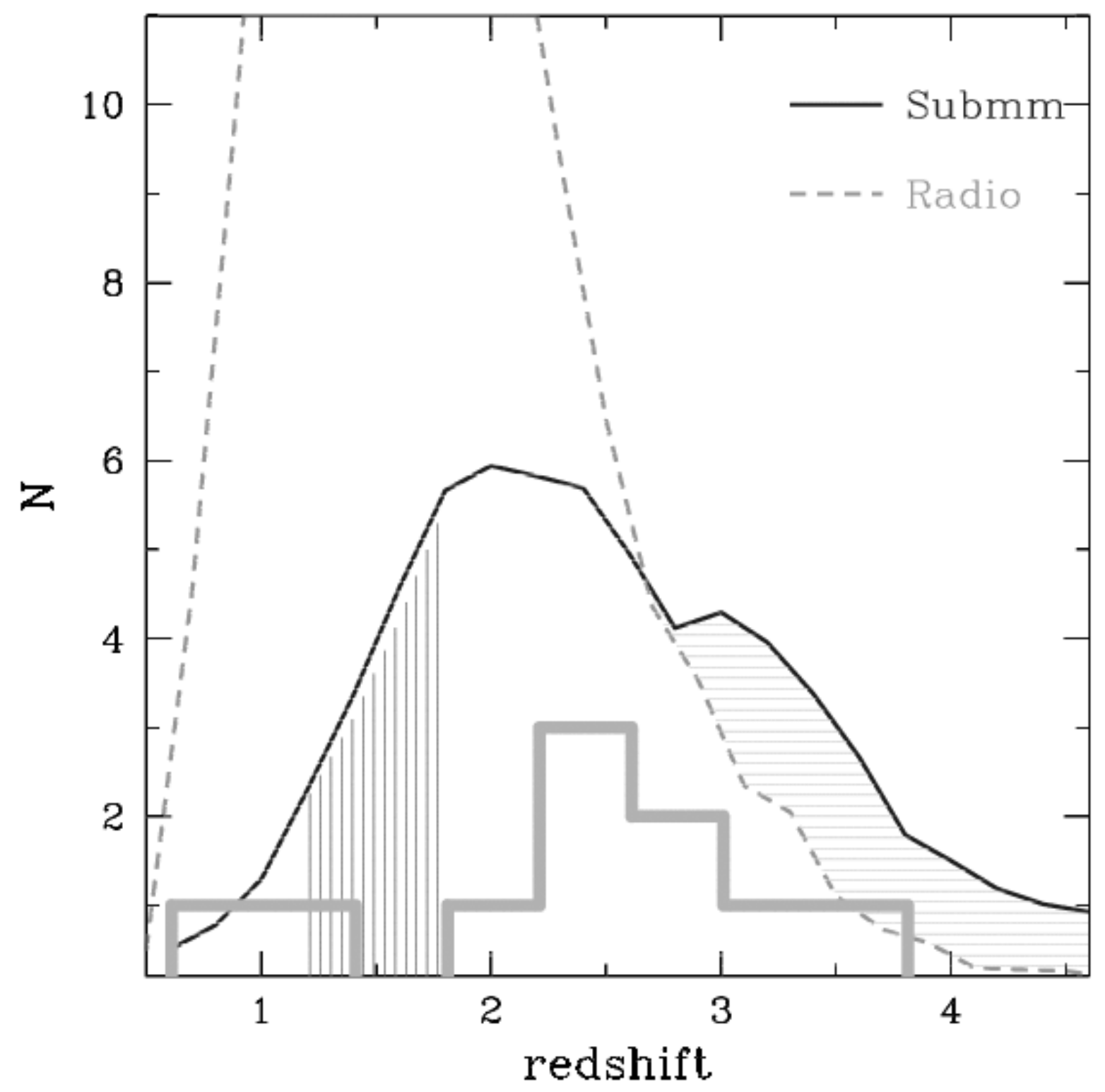

